\title{
Advanced directions of self-control skill individualization of sports activity parameters among young combatants
}

\author{
Olga Bedareva, Galina Kuzmenko*, Tatiana Kim, Elena Levanova \\ Moscow Pedagogical State University, 119991, Moscow, Russia
}

\begin{abstract}
The functions of pedagogical control and self-control are extremely important in modern practice of sports training. They help to optimize training and competitive activity, provide achievements of different qualification athletes. The skills of self-control formation in sports activity parameters among young athletes is an important objective of a coach. In order to achieve it a coach should have a wide spectrum of special competencies. A coach should take into account individual characteristics of his athletes and organize the system of interaction, in terms of which control mechanisms, directed toward own "Self" analysis and regulation would become one of the obligatory factors of young athlete's personality self-consciousness and the condition for his adequate inner and outer world perception. The result of the research held by the authors of the presented article became the essence, content, urgent directions of self-control skill formation in sports activity parameters among young athletes-combatants. We revealed the factors, which condition the ability to control emotional-volitional, cognitive, behavioral spheres of young athletes, taking into account the demands of sports activity and the peculiarities of information perception and processing by them.
\end{abstract}

\section{Introduction}

Sports activity parameters control and self-control is urgent at all stages of sports improvement - from sportshealth improving stage till the stage of higher sportsmanship. Connected with them effectiveness of teaching and upbringing characterizes the increasing adaptive resource of the different qualification athletes competitive behavior. The ability to control inner and outer parameters of the training activity is one of the leading skills. It conditions the quality of mastering the demands of the training process and competitive activity by young combatants. The process of important qualities formation is conditioned by personal participation of a teenager-athlete in mastering the demanded coordinations. They are conditioned by the ability to identify the parameters of activity, compare them with the model characteristics and the expected results, correct the technique, tactics, psycho-emotional state and other things at all stages of motor program realization: from local training or competitive objective till the planned aimed result.

Considering the category "Self-control" in structural- conceptual units of activity is extremely important, as it creates new conditions for urgent self-correction formation by a young athlete the quality of motor actions, functional and psycho-physical state. During the process of sports training it includes the following: readiness self-appraisal to react, self-control over the orienting base of actions, current control over own movements parameters, their key moments realization, the degree of the experienced loads self-appraisal, other constantly alternating acts of self-control. They form necessary precondition for an important component of an expedient control over own behavior. Between the lessons self-control is oriented toward rehabilitating processes self-appraisal, health state analysis, the status of an organism determination and the orientation toward the future trainings. Self-control as the sphere of activity, in our opinion, can be realized only in a wide spectrum of functions: educational; developing (cognitive, psychic development in general, physical qualities and motor-coordinating abilities development); upbringing; health protecting; adaptive; reflexive-estimating; mobilization; controlling (the parameters of activity and behavior optimization); and others.

Structural-conceptual predetermination understanding is provided by defined methodological supporting points for sports training optimization among teenagers-athletes, discussed in the works of N.A. Bernshteyn. They reveal the essence of movement control levels; "sensory corrections", exercise understanding as "activity-based building"; postulate acceptance: ... where at the initial stage of movement mastering "a realized attention can interfere into the details of the movement. They would then evade into the sphere of automatisms" [1]; P.K. Anokhin says about substantiating place of self-control in architectonics of the adaptive behavioral act, where "the results of comparison define further behavior organization" [2]; V.D. Shadrikov - actualizing mental abilities realization through " the results of operation reflexion" and "results parameters reflexion" [3]; M.M. Bogen - defining structural-conceptual content of "motor action technique mastering", revealing "images of feelings" and "the objects of attention localization" [4], L.P. Matveev - characterizing theoretical-methodical and the applied basis of competitive activity technique formation, "conceptual, spatio-temporal, dynamic, rhythmical structure of the movement...in terms of the action"[5]; G.A.

\footnotetext{
${ }^{*}$ Corresponding author: kuzmenkoga2010@yandex.ru
} 
Kuzmenko - specifying the "structure of intellectual resources actualization of a teenager- athlete in solving intellectual problem of sports activity" [6]; T.L. Lugovskikh - reflecting the essence of the quality of educational and sports achievements formation among teenagers-combatants in terms of activity demands integration [7]; V.P. Lukyanenko about the problem of movements formation accuracy [8]; E.A. Levanova with other authors substantiate the importance of a coach cognitive flexibility while differentiating the content of motor actions teaching [9]; S.K. Bagadirova - about the psychological aspects of judo teaching model realization [10]. Scientific-methodical literature analysis concerning the problem of self-control in sports for teenagers and children helped to pay attention to insufficient work over its conceptual units in the sphere of a coach methodical recommendations and a content of the young athlete activity. There is lack of information about complex characteristics of self-control, its content as an independent kind of controllingestimating and correcting activity. It is realized in terms of permanent and/or situational free analysis of its parameters. In this connection the aim of our research work was to define the essence, content, urgent lines of self-control skill formation of sports activity parameters in young athletes-combatants.

\section{Materials and methods}

The research work was held since May, 11, 2019 till March, 15, 2020 on the basis of the "Center of Sport and Education "Sambo-70" and the club of all-styles of karate "Sparta", Moscow, including 30 coaches and 70 12-14 yearold athletes-combatants. They specialize in sambo, judo and karate. The experimental research was characterized by two stages: stating and forming. At the stating stage we defined the problem and content lines of sports activity parameters self-control formation among young combatants. They were the chosen directions of our scientificpedagogical activity. Research methods: scientific-methodical literature analysis, pedagogical observation, semantic, comparative, factorial, cluster analysis.

\section{Results and Discussion}

We defined the urgent parts of a coach's theoretical and applied competency. They demand the reflexion in informative criteria of a young athlete self-control according to sports activity estimation, including hierarchy based theoretical knowledge concerning economic and effective parameters of activity, its image characteristics. The difficulties of self-control realization procedural part, defined by $91,4 \%$ of young athletes, are connected with the fact that coaches don't define the zone of a young athlete responsibility and don't specify the process of the action and activity controlled parameters transfer from the function of pedagogical control into the function self-control, into its permanent, situational aspects, into algorithm based probable behavior. The zone of a young athlete responsibility is formalized, being not developed, if there are no conceptual transformations of an informational idea of a coach, if descriptive characteristics are not added or are not minimized during individually acceptable self-control actualization mastering. Thus, we understand that the sphere of self-control is a sphere of the systemized personality's demands claimed on constructive activity in the specificity of their regulation by a coach, as his activity "reflection" in the activity of a young athlete. Self-control in its generalized notion is a complete picture of self-appraisal criteria of activity process and result, an athlete's feelings and state and in the definite-objective situation their conceptual selectivity and adequacy in terms of the moment during the sides of perception integration and in attributes realization: "I know what I do" and "I feel what I do". At the same time, verbalized picture of self-control attributes "I know what I do" is mainly typical for junior teenagers $(82,9 \%)$. It is typical for them to retell the demands of a coach, only insignificant part of descriptions appear in the structure of movements phases and psycho-physical state "feeling" $(25,7 \%)$. Sensual part of a movement integral image is mainly typical for senior teenagers. It is connected with many factors. Knowledge based component gives place to sensual one. Knowledge is connected with verbalization. Pronouncing provides speed of motor task fulfillment decrease and the retracted formulation of a knowledge- till the level of a mindset or a word-image/word-symbol, describes the experienced muscle-articular sense, dynamic balance and etc. During senior teenage sensual attributes prevail over verbalized conceptual units of self-control. As the tempo of movements increases, the duration of a technical element fulfillment is $0,2-0,5 \mathrm{~ms}$ in a direct training or competitive activity. This makes descriptive characteristics selection difficult and demands additional extra-training time for reflexive analysis of activity parameters organization. In practice the process of self-control actualizes objective $(10,06 \pm 0,751)$, symbolic $(5,53 \pm 0,794)$, signed $(11,18 \pm 0,648)$, representational $(13,47 \pm 1,013)$ thought realization and creativity $(8,77 \pm 0,754)$ (according to J. Bruner test) -young athletes with higher indices have more distinct level of subjective control (internality in the sphere of achievements- $8,3 \pm 0,22$; internality in the sphere of failures- 7,6 $\pm 0,19$ ) and structural-conceptual differentiation of descriptive characteristics - conceptual units. There "a coach's word" has the context: motivating - 32; volitional - 41; emotional - 43; spiritual-moral -40 .

Moreover, self-control is mainly based on clarity, logic, sequence and continuity of visual and kinesthetic row and less on audial characteristics of the action (activity) performing structure taking into account his (her) kinematic and dynamic parameters, if we speak about teaching the technique of a motor action. A teenager can easily master spatiotemporal characteristics of a movement in comparison with dynamic parameters. They are conditioned by the ability to realize muscle efforts differentiation and close to maximal and maximal effort alternation with less expressed phases in an integral motor action. As a visual row lies on the surface of material perception a coach pays attention to spatio and temporal localization of the main points. Emotional-sensual sphere is specific and in these emotions and feelings description for one and the same fragment of an action 70 teenagers-athletes present till 8 typical descriptions. It means 
that self-control helps to regulate the difference between modal canals of the definite information perception. Pedagogical analysis of 18 educational-training lessons content, held by the coaches ( $\mathrm{n}=9$ ), helps to set some disintegration in the exercise description: great preferences for spatio-temporal parameters $(82,8 \%)$ and less for dynamic efforts making $(22,8 \%)$.

The attributes of self-control of 13-14 year-old teenagers' activity rationalization include adaptation to the opponent's behavior. There great importance has the opponent's activity control. During the expected results of sports activity formation it is important to understand owing to what logic the system of demands claimed on motor action is organized. We revealed spatial peculiarities of teenagers' notions accumulation concerning the technique of motor action, its essence understanding, in which descriptive characteristic is built from: the center of body masses; the final joints, which reflect amplitude characteristics of movements; movement parameters conjugation; orientation toward the work coordination in joints in terms of restricting the degrees of freedom; synchronization or an optimizing delay of the similar and not similar (sides) parts of the body; combinations of the full amplitude of one parts of the body with a partial amplitude of other parts. During self-control of motor actions technique formation it is important to understand two moments: "What is the space of a relevant zone of self-control?"; "What technical elements and demands claimed on their fulfillment are out of self-control zone?". We defined the variants of the response actions as a resulting reaction to self-control during motor action technique and its parameters formation: 1) direct influence; 2) reversal influence, when the formed parameter of the technique lies beyond the limits of self-control zone and the initial action forms the condition for a successful realization. Organizing educational-training process a coach should understand the zone of self-control effect localization from the position of the relevant and proximal development zones during self-control criteria of different order determination. In this connection an important role belongs to the form and content of professional communication, in which a coach not only selects a significant word-demand, but also predicts and defines possible influence of this word on the attributes of self-control formation. It makes the importance of professional competency skills development in formulating correct and clear to a young athlete attributes of activity quality. They reflect sensual results of motor and intellectual activity. Pedagogical analysis of the motor actions technique formation conditions helps to set the presence of a strong correlational connection between the following: the level of mastering technical elements (1) and the availability of the descriptive characteristics (2), ( $\mathrm{r}=0,72)$; (1) and sensual descriptive characteristics presence (3), $(r=0,64)$. Conjugation of the controlled parameters with the parameters at the first stages of not realized motor activity and later with the activity in terms of the realized mastering its characteristics helps to control not only the tempo of mastering educational material in sport, but also define the reference positions in a wide spectrum of abilities and skills, as the key positions of self-control. They provide the set activity parameters formation in young combatants.

Classical scheme of educational-training lesson with the main orientation toward the technique teaching or toward physical qualities and motor-coordinating abilities upbringing is characterized by close conjugation of these objectives, when technical elements resulting mastering happens on the background of the increasing load of different orientation (physical and psychic). Pedagogical competency of a coach is characterized by the ability to synchronize the technique of movement with its descriptive characteristics, where there are the tendencies of the activity based picture formation, as its content includes not the components, said by a coach, but realized by a young athlete, analytically and synthetically mastered by him information. The recommended algorithm of teaching an action during self-control realization (based on the parameters self-analysis, self-correction) is the following: video of a movement; slow reproduction of an action; an athlete's description of a visual row of a movement during "not felt", information free phases analysis; structural-conceptual addition of the exercises fulfillment information-sensual picture. In terms of a high motor density and the absence of rest intervals (between the series of exercises fulfillment) the tasks for feelings reflexion, movement parameters analysis- there is the amount decrease of the realized sensual characteristics of the mastered and developed motor actions. We revealed not similar picture of self-control perception among young combatants, where the fulfillment is connected with the following: 1) operations - with "feel in details" mindset, 2) actions - "make everything to feel"; 3) motor activity - in terms of each repeated attempt- synchronize, aim at "the relevant phase of a movement by the sense of a feeling". In this connection the skills of self-control realization in different time parts of a movement fulfillment "from a motor operation to motor activity" gains maximal importance. Generally adopted logic of action mastering first as an independent unit and further in the structure of activity, in selfcontrol is the contrary. In the activity of a young athlete in the hierarchy of the greatest difficulty is self-control: 1) operations themselves; 2) operations in the structure of a motor action; 3) operations in the structure of a cyclic motor activity. It is connected with the ability to synchronize thought and the operation (or an action), organize, adapt the attributes of self-control to a rhythmic structure of a movement. The results analysis of the thought realization quality self-assessment in action or an operation (according to the estimating scale 1-3 points) by combatants helps to state positive correlational dependence with a high and the average inclination for a risk $(r=0,76)$ and "a quick time flow", where an individual minute of a young athlete - 56-57 s and less; inclination for synthetical character of thinking. It conditions the necessity for "slow", analytical teenagers set smaller tempos of stimuli presentation, as teaching in sport is connected with the factor of mastering information, its interiorization in activity.

Young athletes with the prevailing "synthetical character" and "analytical character" of thinking have different logic schemes of mastering attributes of movement and structural components of his self-control: the first have the following logic of mastering material "from a feeling to the parameters of a movement stating"; the second - from systematization and hierarchy of activity demands- to feeling the specificity of technically correct movement fulfillment. 
Self-control as a specifically directed activity is actualized when a teenager realizes cause-effect relationship, conditioned by completeness of characteristics, but at the same time, self-control criteria form hierarchy according to the principle "necessary and sufficient", choosing from the whole spectrum of characteristics the most significant. We defined that self-control parameters in the structure of an action or activity are distanted from each other and don't present two successive and connected phases of movement, as self-control presents thinking activity. It is very often verbalized at the stage of mastering and makes movement slow. Pedagogical analysis of teen-agers description helps to characterize the controlled parts of sports activity as the phases of direct control and tracking (which violate the tempo of actions) and the phases of an indirect tracking. They intensify rhythmic structure of activity. During technical elements mastering it is necessary to pay attention to the phase. It is before the main mastered (for example, not at a swing, but at an active, amplitude returnable movement; at the beginning of the positive speeding up phase).

Descriptive characteristic of a technical element, technical-tactical connection helped to define the prevailing cross connection of sensual zone attributes and the zone of the applied knowledge. The teenagers mentioned the peculiarity: if everything is described by sensual words-symbols, or notions of the applied knowledge we lose the sense of an integral action fulfillment tempo. The difference between the descriptive visual and kinesthetic characteristics of motor activity actualizes reflexive analysis of a motor action effective phases sensual attributes fulfillment. Pedagogical observation helped to define that an activity based picture of kinesthetic (sensual) images during technical elements fulfillment is more effectively created in terms of two-three variants of the movements comparison performing structure: effective and less effective parameters of actions - teaching on the basis of sensual feelings comparison in terms of effective movement and the movement "with a mistake". In this connection it is pedagogically important to specify the zones of self-control taking into account the phases of motor action, which young athletes characterize as: "don't feel" and "don't understand". Creating the finished picture of an integral image of a motor action helps to organize an integral group of characteristics, varying which: from not fixed in consciousness descriptive mindsets to not used earlier sensual images, we connect a dynamic picture of self-control markers in terms of technique parameters optimization. The expected results of movement, its essence understanding is different among different typological groups: from a feeling to the parameters of movement understanding; from the definite demands to the feeling identification.

The problematics of self-control formation in young combatants is in the fact that sensual spheres of a coach and an athlete are different. A coach from the position of a great motor (sensual) experience claims rational demands on economic and effective technique. If conditional and coordinating abilities allow young athlete feel this optimal movement, a young athlete will feel the set feeling. Self-control parameters individualization is in the fact that young combatants select urgent for a moment, available for mastering criteria of effectiveness from the whole spectrum of the presented demands-descriptions. Pedagogical analysis of self-descriptions concerning the effectiveness of action loss helps to underline that the duration of self-control phase corresponds and is shorter than the successful part of the activity. Self-analysis of self-control realization during dynamic balance keeping helps to mention that self-control is fixed not till the moment of complete balance loss, but till the parts of the body imbalance in the statics or in dynamics.

Logically organized program of self-control is based on the actions sequence of a coach and an athlete taking into account the following parts: intellectual- which conditions the object of self-control determination and acceptable space of variation by the demands complex in the activity. It is realized either at theoretical intellectual level (which anticipates theoretical intellect actualization with the variants of actions algorithmization) or at the level of the existing motor experience of a parallel possible prediction using practical intellect on the background of defining the width of attention zone or earlier realized knowledge; structural-conceptual. It conditions the content and localization of demands claimed on the controlled parameters of an operation, action, sports, volitional and regulating one (psychic activity mobilization for the definite quality criteria holding in the sphere of self-control, permanent, current control and action combination with the expected result). Defined by us leading factors of self-control formation in practice of sport (table) characterize key demands claimed on pedagogical interaction organization in the system "coach-athlete".

Table 1. Leading factors (LF) of self-control skills formation of young combatants' (12-14 year-old (y)) activity

\begin{tabular}{|c|l|c|c|}
\hline \multirow{2}{*}{$№$} & The content of factors, which reflect effective ways of self-control formation over sports activity & \multicolumn{2}{|c|}{ LF } \\
\cline { 3 - 4 } & \multicolumn{1}{|c|}{1 (SA) parameters in young combatants } & 13 y & $13-14 \mathrm{y}$ \\
\hline 1 & specificity of movement (M) self-control criteria & $\mathbf{0 , 7 9 5}$ & 0,232 \\
\hline 2 & $\begin{array}{l}\text { continuity (with the directed pedagogical effects delay and advance) in pedagogical control and } \\
\text { self-control of sports training }\end{array}$ & $\mathbf{0 , 7 3 0}$ & 0,384 \\
\hline 3 & the content of pedagogical control of sports training transfer into the sphere of self-control & 0,515 & $\mathbf{0 , 9 0 8}$ \\
\hline 4 & $\begin{array}{l}\text { intellectual potential actualization in terms of the educational-training objectives content and the } \\
\text { attributes of self-control conjugation }\end{array}$ & 0,516 & $\mathbf{0 , 9 2 2}$ \\
\hline 5 & accents of pedagogical recommendations and motor images matching & $\mathbf{0 , 7 5 0}$ & $\mathbf{0 , 7 2 2}$ \\
\hline 6 & pedagogical adjustment to the content of an individual picture of self-control & $\mathbf{0 , 7 4 4}$ & $\mathbf{0 , 8 3 2}$ \\
\hline 7 & definiteness of a young athlete responsibility zone in the system of SA control & $-0,297$ & $\mathbf{0 , 8 7 8}$ \\
\hline 8 & $\begin{array}{l}\text { substantial connection of rational mindsets for the future activity structure self-control with the } \\
\text { motivating, volitional, emotional, spiritual-moral essences of a coach's communication }\end{array}$ & 0,647 & $\mathbf{0 , 8 9 2}$ \\
\hline 9 & individually acceptable succession of self-control zones actualization & 0,681 & $\mathbf{0 , 7 4 5}$ \\
\hline 10 & $\begin{array}{l}\text { substantial selectivity, adequacy of situation self-control criteria in terms of perception and } \\
\text { activity integration ("know and feel, what I do") }\end{array}$ & $\mathbf{0 , 8 8 0}$ & 0,451 \\
\hline 11 & the formation level of knowledge based and sensual parts of an integral M image & $\mathbf{0 , 7 5 8}$ & $\mathbf{0 , 9 0 7}$ \\
\hline 12 & opponent's activity control on the basis of personal behavior self-control & $-0,638$ & $\mathbf{- 0 , 7 8 1}$ \\
\hline
\end{tabular}




\begin{tabular}{|c|l|c|c|}
\hline 13 & definiteness of different order self-control criteria & 0,364 & $\mathbf{0 , 9 0 7}$ \\
\hline 14 & completeness of an exercise fulfillment information-sensual picture & $-0,871$ & 0,529 \\
\hline 15 & adequacy of self-control in terms of M objective realization complication & 0,311 & $\mathbf{0 , 8 2 0}$ \\
\hline
\end{tabular}

Categoriality of teen-agers' thoughts demands mastering another kind of perception in achieving the controlled parameters. Self-control over personal activity is seen in predominance over the opponent or the existing success of technical elements fulfillment: "led..."; "held..."; "controlled ...". Not successfully fulfilled motor operation, action are estimated negatively as not controlled and not realized. In this connection, actions parameters detailing helps to define the zone of the mistake localization and further realize their directed mastering. Unregulated amount of attempts decreases the following: responsibility before the movement fulfillment $(\mathrm{r}=-0,73)$; mobilization efforts realization (according to self-assessment (SA) results), ( $\mathrm{r}=-0,68)$; creates the notion of an endless amount of opportunities, when there is mobilization abilities "here and now" realization in sports competition. In the experiment we accepted the mindset for activity "with one attempt" with the line of teen-ager's thinking correction "directed toward one more attempt". Taking into account high SA - one attempt; in terms of the average SA- two attempts; in terms of a low SAthree attempts. The coaches used two ways of results calculation:

+1 successful action; +2 and further; or stated not successful attempts- from the initial 6 points $-\ll-1$ point for a mistake»; «-2» and further, which conditioned permanent self-control over activity parameters. Pedagogical activity of a coach, based only on taking into account age related peculiarities of free control realization out of real demands of competitive activity connection, creates the case of an athlete's consciousness "separation" from the demands of a cognitive, physical development, physical (conditional, coordinating) readiness, competitive traits of personality development and formation in activity.

Questionnaire survey among 70 12-14 year-old combatants, the representatives of karate ( $\mathrm{n}=25)$, judo (23), sambo (22) helped to characterize the specificity of a coach professional behavior: 1) with high, maximum strict system of demands claimed on a teen-ager's activity as the formed part of a personality $(54,3 \%) ; 2$ ) with a high and maximum strict system of demands. They achieve pedagogical effect of movements accuracy on the background of fear. It provides efforts mobilization $(35,7 \%)$; 3) with highly-differentiated, detailed system of demands according to the quality of motor actions fulfillment in terms of multiple mastering movement parameters "for its conceptual characteristics understanding" $(25,7 \%)$; 4 ) not having the ability to present the system of demands in details and clear $(37,1 \%) ; 5)$ not sufficiently motivated coaches for the quality of movement teaching $(34,3 \%)$. It was also revealed that some teen-agers had the experience of communication with different types of trainers, others decided to conceal drawbacks- trainer's incompetence, not answering the questions. However, the necessity to improve coach's professional competency is evident, as only $25,7 \%$ of coaches consider it important to form the realized attitude to the studied motor action. Considering the conditions of competition helps a teen-ager to master activity demands consequently on the background of widening the zones of personal and collective responsibility. In accordance with a young athlete's motor activity detailing a coach tries to organize his intellectual activity in mastering urgent parameters of sports activity on the basis of analysis, comparison, summarizing, feeling in the training and in competition. It is obvious that self-control function is connected with self-analysis, self-correction, self-management, self-development with the enriched worldview of a teen-ager, where only he can achieve the definite achievements, learning how to form the lines of purpose setting in sports activity, with personal maturity. The dynamics of conceptual supports in selfcontrol formation in young combatants reflected axiological potential use of the self-control studied components,. They became the main in achievements indices improvement. The quality of mastering educational material of educationaltraining lessons improved from $3,8 \pm 0,52$ till $4,2 \pm 0,45$ points.

\section{Conclusions}

1) Successful realization of self-control function is possible in terms of the following: the role of a young athlete's personality increase in the system of pedagogical control, the value of a teen-ager's estimating thoughts increase; a young athlete's opinion respect; the results of self-assessment and pedagogical assessment equalization; pedagogical control realized by a coach over professional destructions, in which self-control of a young athlete is realized on the basis of pedagogical reproach and insult fear. Theoretical training importance is also evident. If the subject of the future activity is not defined beforehand, a coach and a young athlete think using different categories - "knowledge" and "ignorance". 2) Taking into account the leading factors of self-control formation helps to optimize the process and results of pedagogical activity in the system "coach-athlete". Basic clusters in self-control formation in 12-13 year-old combatants are the following: a) continuity in the content of pedagogical control development and the content of selfcontrol over sports training; b) matching the accents of pedagogical recommendations and motor images; c) mastering tempo self-control, which intensifies rhythmic structure of an action. Basic clusters for 13-14 year-old combatants: a) "the content of pedagogical control of sports training transfer into the sphere of self-control" and "intellectual potential actualization in terms of the educational-training objectives content and the attributes of self-control conjugation"; b) "opponent's activity control on the basis of personal behavior self-control" and "the definite character of the mistake localization zone". Positive dynamics of mastering the technique of motor actions fulfillment proved the effectiveness of the revealed and realized promising lines of self-control formation over sports activity parameters.

\section{References}


1. N.A. Bernshteyn, About the dexterity and its development (Physical culture and sport, Moscow, 1991)

2. A.S. Kuznetsov, Z.M. Kuznetsova, Russian Journal of Physical Education and Sport, 14(4), 5-7 (2019)

3. V.D. Shadrikov, Mental development of a person (AspectPress, Moscow, 2007)

4. M.M. Bogen, Motor actions teaching (Physical culture and sport, Moscow, 1985)

5. L.P. Matveev, General theory and its applied aspects (Publishing house "Lan”, Saint-Petersburg, 2005)

6. G.A. Kuzmenko, Concept of intellectual development of teen-agers in sports for children and teen-agers (MPSU, Moscow, 2017)

7. T.N. Lugovskikh, Scientific notes of P.F. Lesgaft University, 3(157), 204-209 (2018)

8. V.P. Lukyanenko, Theory and Practice of Physical Culture, 1, $43-45$ (2018)

9. G.A. Kuzmenko, T.K. Kim, E.A. Levanova, V.S. Sherin, Bulletin of Tomsk State University, 440, 167-174 (2019)

10. S.K. Bagadirova, Theory and Practice of Physical Culture, 6, 23-25 (2020) 